\title{
Fucoidan Immobilized at the Surface of a Fibrous Mesh Presents Toxic Effects over Melanoma Cells, But Not over Noncancer Skin Cells
}

Catarina Oliveira, Ana I. Soares, Nuno M. Neves, Rui L. Reis, Alexandra P. Marques, Tiago H. Silva, and Albino Martins*

Cite This: Biomacromolecules 2020, 21, 2745-2754

ABSTRACT: The use of fucoidan, a marine-origin bioactive polymer, is herein proposed as a component of an innovative and effective strategy against melanoma, one of the most aggressive skin cancers. First, fucoidan antitumor activity, in its soluble form, was assessed presenting increased cytotoxicity over melanoma cells when compared to human dermal fibroblasts and keratinocytes. After this antitumor activity validation and trying to develop a more targeted and local strategy aiming to diminish the cytotoxic effects over noncancer cells, fucoidan was immobilized at the surface of an electrospun nanofiber mesh (NFM_Fu), envisioning the development of a therapeutic patch. The maximum immobilization concentration was $1.2 \mathrm{mg} \mathrm{mL}^{-1}$, determined by the Toluidine Blue Assay and confirmed by XPS. Furthermore, NFM_Fu is more hydrophilic than NFM, presenting a contact angle of $36^{\circ}$, lower than the $121^{\circ}$ of the control condition. NFM_Fu was able to reduce human melanoma cell viability by $50 \%$ without affecting human dermal fibroblasts and keratinocytes. Taken together, these results set the basis for a valuable approach for melanoma treatment.

\section{INTRODUCTION}

Current treatment modalities for cancer do not achieve the ideal therapeutic outcomes due to severe side effects experienced by cancer patients. ${ }^{1}$ In this sense, alternative approaches are required and, between the possibilities, there is an increasing interest in the use of natural compounds from marine resources as biologically active products. Brown algae are a source of polysaccharides that may present several biological responses. ${ }^{2-4}$ Among these marine origin materials, fucoidan has attracted enormous interest in the last recent years. ${ }^{5}$ Fucoidan is a polysaccharide consisting of a sequence of sulfated fucose residues, together with other sugars, namely, uronic acids, with a chemical structure depending on the specie and extraction parameters, among other factors. ${ }^{6}$ Different physicochemical properties, such as molecular weight, carbohydrates composition, sulfation degree, and pattern along the fucoidan backbone, have been related to its biological activities, ${ }^{6-8}$ such as antitumor, antiangiogenic, and anti-inflammatory. ${ }^{5,9}$ Particularly, fucoidan has been recognized as a potential antitumor agent for different types of cancers, like breast, lung, colon, and melanoma. ${ }^{10-14}$

Melanoma represents about $1 \%$ of all skin tumors. However, it is the most aggressive and deadliest form of skin cancer, having a very poor prognosis when it becomes metastatic. ${ }^{15-17}$ Risk factors for melanoma include sunburns during childhood, genetic family history, and intermittent exposure to strong sunlight. ${ }^{18}$ Current treatment modalities may be surgical resection, chemotherapy, immunotherapy, and targeted therapy, depending on the tumor characteristics (size, site, and genetic profile). ${ }^{19,20}$ To improve survival rates, the combination of different therapies is often recommended. The lack of specificity to tumor cells is one of the major limitations of current therapies that may result in adverse effects and reduced efficiency. ${ }^{21}$ Trying to overcome these limitations, the need for testing alternative strategies and compounds, offers interesting possibilities. ${ }^{22}$

Fucoidan has been reported to inhibit melanoma development and progression, namely, in its soluble form. Crude commercial fucoidan extracts from Fucus vesiculosus and Sargassum sp. showed that, despite the demonstrated cell growth inhibition at low dosages, both extracts presented very similar cytotoxic profiles over melanoma cells. ${ }^{23}$ In another

Received: April 2, 2020

Revised: May 14, 2020

Published: May 18, 2020 
attempt, similar extracts were studied, with a comparable antiproliferative trend being crude commercial fucoidan more cytotoxic than the extracted fucoidan at higher doses. ${ }^{24}$ It was also demonstrated that fucoidan reduced the proliferation of melanoma cells and melanin production in a dose-dependent manner, producing alterations in the cells' morphology. ${ }^{25}$ The antitumor activity of fucoidan extracted from Undaria pinnatifida and Fucus evanescens was evaluated over different human cancer cell lines, namely, colon, breast, and melanoma. $^{13}$ No cytotoxic effects were observed over a noncancer mouse epidermal cell line, whereas more pronounced growth inhibition was observed for breast and melanoma cells. A recent study evaluated the toxicity of fucoidan from Fucus vesiculosus over B16 murine melanoma cells, showing growth inhibition by regulating specific protein/ enzyme expression levels. ${ }^{14}$ However, as previously reported by our group, not all fucoidan extracts present this interesting and promising antitumor activity, relating its biological activity with its chemical structure. Indeed, some fucoidan extracts may present antitumor properties against some (not all) cell lines, as well as having different cytotoxic concentrations. ${ }^{7,26}$ In fact, despite the previous reports on the cytotoxic effect of different fucoidan extracts over melanoma cells, another commercially available fucoidan from Fucus vesiculosus was evaluated over five different uveal melanoma cells lines not showing antitumorigenic effects. ${ }^{27}$

Based on the discrepancy of published results, in this study, we evaluated and validated the toxicity of soluble fucoidan not only over human melanoma cells (WM-115 cell line), but also over primary dermal fibroblasts and keratinocytes as models of the main cell types characterizing the tumor microenvironment. An alternative approach to face melanoma is herein proposed, aiming to decrease the cytotoxic effects over noncancer cells. A tailored nanofiber mesh functionalized with fucoidan (NFM_Fu) was developed, envisioning a future skin patch to treat melanoma in a more local, precise, and effective way, as well as a complementary treatment after tumor excision.

\section{EXPERIMENTAL SECTION}

2.1. Materials. Polycaprolactone (PCL; batch number: MKBP7389 V; $\left.M_{\mathrm{w}}=70000-90000\right)$, chloroform, N,N-dimethylformamide (DMF), 1,6-hexanediamine (HMD), and fucoidan from Fucus vesiculosus (Fu; batch number: SLBP3196 V; $M_{\mathrm{w}}=107800$ ) were purchased from Sigma-Aldrich and used as received.

2.2. Production of Nanofibrous Meshes. A $15 \%(\mathrm{w} / \mathrm{v})$ PCL solution was prepared with an organic solvent mixture of chloroform and DMF at a 7:3 ratio. The PCL solution was electrospun by applying a voltage of $11.1 \mathrm{kV}$, a needle tip to ground collector distance of $18 \mathrm{~cm}$, and a flow rate of $1 \mathrm{~mL} \mathrm{~h}^{-1}$. After the complete processing of $1 \mathrm{~mL}$ of PCL solution, the nanofibrous mesh (NFM) was allowed to dry for 1 day. ${ }^{28}$ These processed NFMs were cut into samples of 1 $\times 1 \mathrm{~cm}^{2}$ for further assays.

2.3. Ultraviolet-Ozone Irradiation and Aminolysis. For the activation of the NFM, an ultraviolet-ozone (UV-ozone) cleaner system was used (ProCleaner 220, Bioforce Nanoscience). Both sides of the electrospun NFMs were exposed for $90 \mathrm{~s}$ to UV-ozone irradiation. After this surface activation, amine groups $\left(-\mathrm{NH}_{2}\right)$ were inserted at the surface of NFM by immersion in a $1 \mathrm{M}$ HMD solution for $1 \mathrm{~h}$ at $37^{\circ} \mathrm{C}$. Finally, the functionalized NFM were washed $3 \times$ with PBS. ${ }^{29}$

2.4. Fucoidan Immobilization of NFM. Fucoidan was dissolved in $0.1 \mathrm{M} \mathrm{NaCl}$ at different concentrations ( $\mathrm{Fu} \mathrm{2.5,} \mathrm{Fu} \mathrm{5,} \mathrm{Fu} \mathrm{7.5,} \mathrm{and}$ Fu $10 \mathrm{mg} \mathrm{mL}^{-1}$ ), and $200 \mu \mathrm{L}$ of each solution was added over functionalized NFM in a 24-well plate. This reaction was performed over $8 \mathrm{~h}$, and after that, the solutions were removed and the biofunctionalized NFM were left to dry overnight. In the following day, NFM were washed twice. All steps were performed under sterile conditions.

2.5. Quantification of Immobilized Fucoidan: Toluidine Blue Assay. Toluidine Blue (TBO) was used to quantify the amount of fucoidan immobilized into each NFM. ${ }^{30}$ Each sample was immersed in $500 \mu \mathrm{L}$ of TBO solution $(0.1 \mathrm{M} \mathrm{HCl}, 20 \mathrm{mg} \mathrm{NaCl}$, and $4 \mathrm{mg}$ toluidine blue $\mathrm{O}$ chloride) for $4 \mathrm{~h}$ at room temperature. The TBO solution was removed, and the biofunctionalized NFMs were washed until all unreacted TBO solution was removed. Afterward, the biofunctionalized NFMs were immersed in $500 \mu \mathrm{L}$ of a solution containing $0.1 \mathrm{M} \mathrm{NaCl}$ and ethanol (1:4) for complete decoloration. The amount of TBO was assessed by measuring the absorbance of the supernatant at $530 \mathrm{~nm}$ using a microplate reader. The amount of fucoidan immobilized at the surface of each NFM was calculated from a standard curve established with fucoidan solutions at different concentrations ( $\mathrm{Fu} \mathrm{2.5,} \mathrm{Fu} \mathrm{5,} \mathrm{Fu} \mathrm{7.5,} \mathrm{and} \mathrm{Fu} 10 \mathrm{mg} \mathrm{mL}^{-1}$ ). In this sense, after incubating fucoidan with $\mathrm{TBO}$, the solutions were centrifuged and the unbound $\mathrm{TBO}$ was removed. The pellet was then resuspended with the above-mentioned $0.1 \mathrm{M} \mathrm{NaCl}$ and ethanol solution and read at $530 \mathrm{~nm}$.

2.6. Contact Angle. Surface hydrophilicity of NFM and NFM_Fu was measured as the static contact angle of a standard liquid (ultrapure water, $3 \mu \mathrm{L}$ ), at room temperature, using Contact Angle Equipment (OCA 15plus equipment, Germany and SCA-20 software). During every determination, a motor driven syringe was used to deposit a drop of liquid over the NFM surface. Measurements were recorded for each sample, and the determinations were performed in triplicate.

2.7. XPS Analysis. Analysis of the samples was performed using a Kratos Axis-Supra instrument controlled with ESCApe software. Due to the nonconductive nature of the samples, it was necessary to use a coaxial electron neutralizer to minimize surface charging. The XPS measurements were carried out using monochromatic $\mathrm{Al}-\mathrm{K} \alpha$ radiation $(1486.6 \mathrm{eV})$. Photoelectrons were collected from a takeoff angle of $90^{\circ}$ relative to the sample surface. The measurement was done in a Constant Analyzer Energy mode (CAE) with a $160 \mathrm{eV}$ pass energy and $15 \mathrm{~mA}$ of emission current for survey spectra and $40 \mathrm{eV}$ pass energy for high resolution spectra, also using an emission current of $15 \mathrm{~mA}$. Charge referencing was done by setting the lower binding energy C 1 s photo peak at $285.0 \mathrm{eV} \mathrm{C} 1$ s hydrocarbon peak. ${ }^{31}$ The chemical composition of the samples was examined by XPS surface measurements. The $\mathrm{C} 1 \mathrm{~s}, \mathrm{O} 1 \mathrm{~s}, \mathrm{~S} 2 \mathrm{p}$, and survey spectra were recorded using a Kratos Axis-Supra instrument. The residual vacuum in the X-ray analysis chamber was maintained at around $4.5 \times 10^{8}$ Torr. The samples were fixed to the sample holder with double-sided carbon tape.

2.8. Biological Assays. 2.8.1. Cell Culture. Human melanoma cells (WM-115 cell line, ATCC) were cultured in Eagle's Minimum Essential Medium (EMEM 30-2003, ATCC), supplemented with $10 \%$ FBS (Alfagene), 1\% pen/strep (100 U/100 $\mu \mathrm{g} \mathrm{mL}^{-1}$; Life Technologies), and incubated at $37{ }^{\circ} \mathrm{C}$ in a humidified $5 \% \mathrm{CO}_{2}$ atmosphere. Human dermal fibroblasts (hDFs, Gibco) were cultured in Medium 106 (Gibco) supplemented with 10\% FBS (Alfagene), Pen/Strep (100 U/100 $\mathrm{g} \mathrm{mL}^{-1}$; Life Technologies), and 2\% Low Serum Growth Supplement (Life Technologies). Human keratinocytes (hKCs) were isolated as previously published by our group. ${ }^{32}$ hKCs were cultured in Keratinocyte-SFM (Life Technologies), supplemented with $1 \%$ Pen/Strep (100 U/100 $\mathrm{g} \mathrm{mL}^{-1}$; Life Technologies). The medium was changed every two days until cells reached confluence.

2.8.2. Cell Seeding for a Soluble Fucoidan Test. Melanoma and normal skin cells were cultured at a density of 15000 cells and were cultured in 24-well plates. The cells were left to adhere for $4 \mathrm{~h}$, and after that, fucoidan extracts were added to adherent cells. Fucoidan extracts were dissolved in the corresponding culture medium at different concentrations (i.e., $0.25,0.5,0.75$, and $1 \mathrm{mg} \mathrm{mL}^{-1}$ ). For all the assays, a positive control was performed without fucoidan. Each 


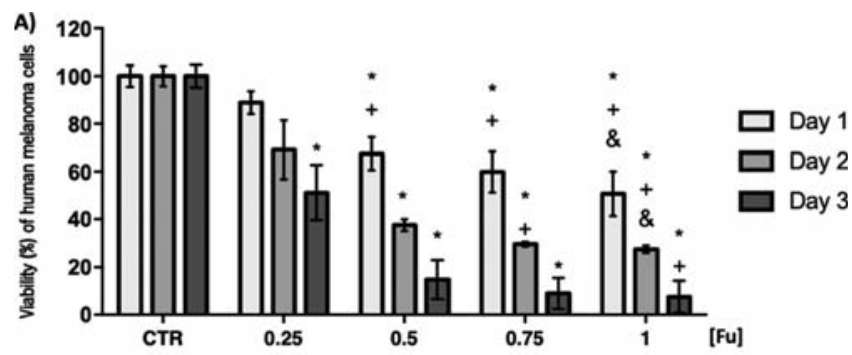

2.8.4. Viability Assays. The metabolic activity was determined by the MTS assay (CellTiter 96 AQueous One Solution, Promega). Briefly, at days 1,3 , and 7 , the culture medium was removed and the samples were rinsed with sterile PBS. A mixture of culture medium and MTS reagent (5:1 ratio) was added to each condition as well as to the negative control comprising no cells. Samples were left to incubate for $3 \mathrm{~h}$ at $37{ }^{\circ} \mathrm{C}$ in a humidified $5 \% \mathrm{CO}_{2}$ atm. Thereafter, the absorbance of the MTS reaction medium from each sample was read in triplicate at $490 \mathrm{~nm}$ (Synergy HT, BioTEK).

2.8.5. Scanning Electron Microscopy Analysis. The morphology and distribution of cells seeded on top of the NFM_Fu or NFM CTR were analyzed by Scanning Electron Microscopy $(\mathrm{SEM})$. At each time point $(1,3$, and 7 days), samples were collected and fixed with $2.5 \%$ glutaraldehyde. After fixation, samples were washed with PBS and dehydrated with increasing concentrations of ethanol, until $100 \%$ was reached. Samples were then left to dry overnight. In the following day, all samples were vacuum-coated with a platinum mixture and observed at the SEM (JSM-6010 LV, JEOL, Japan). Photographs at $\times 150$ and $\times 1000$ magnifications were obtained.

2.9. Statistical Analysis. Statistical analysis was performed using Graph Pad Prism Software. Differences between the testing conditions were analyzed using the nonparametric test (KruskalWallis test), since the data did not follow a normal distribution and a $p<0.05$ was considered significant. Data are presented as mean \pm standard deviations.

\section{RESULTS}

3.1. Cytotoxicity Effects of Soluble Fucoidan over Human Melanoma, Keratinocytes, and Dermal Fibroblasts. Increased toxic effects of fucoidan over human melanoma cells started to be observed for $0.5 \mathrm{mg} \mathrm{mL}-1$ concentration and above. At day 3, melanoma cells' viability reached values below $20 \%$ for those fucoidan concentrations (Figure 1A). Regarding noncancer cells, namely, over primary human keratinocytes, fucoidan presented significant toxicity at day 2 for $0.25 \mathrm{mg} \mathrm{mL}^{-1}$ and above, presenting a toxicity between 40 and $55 \%$ at day 3 when compared with the CTR being dose-independent (Figure 1B). For dermal fibroblasts, fucoidan presented significant cytotoxicity for days 2 and 3 when comparing the different concentrations, with the control presenting a maximum toxicity around $25-30 \%$, which was also independent of the dosage (Figure 1C).

3.2. Quantification of Fucoidan Immobilized on NFM. Different concentrations of fucoidan were immobilized at the surface of NFM until its saturation concentration $(10 \mathrm{mg}$ $\mathrm{mL}^{-1}$, according to the manufacture information). After optimizing a standard curve, the different concentrations were calculated, and it was observe that, by increasing the were used in three independent assays $(n=3)$.
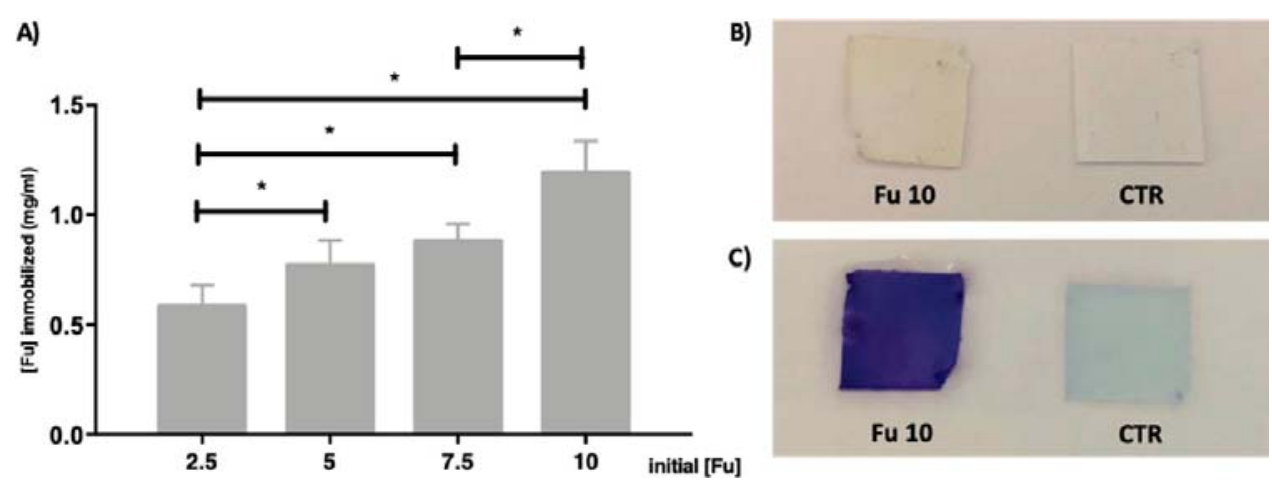

Figure 2. Quantification of fucoidan immobilized on the NFMs (A). Change of color after fucoidan immobilization (B) and after Toluidine Blue assay (C). 


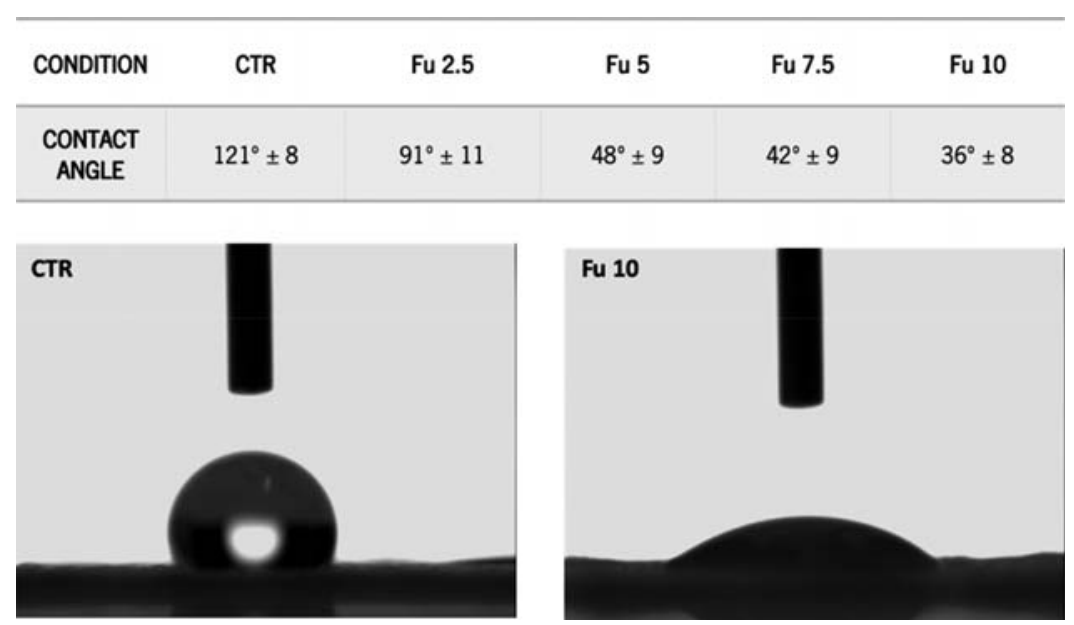

Figure 3. Water contact angle measurements for the NFMs without and with fucoidan immobilized at different concentrations.

initial concentration of fucoidan, higher amounts are immobilized. The maximum immobilization capacity was around $1.2 \mathrm{mg} \mathrm{mL}^{-1}$ for Fu10, twice the concentration of Fu2.5 with a minimum immobilization of around $0.6 \mathrm{mg} \mathrm{mL}^{-1}$ (Figure 2A). The lower concentrations Fu5 and Fu2.5 are the ones with higher immobilization percentages when compared to the initial concentration, $16 \%$ and $26 \%$, respectively. Fu 10 and Fu7.5 have similar immobilization percentages of around $12 \%$. Nevertheless, these are the two concentrations with more fucoidan immobilized on the NFM (Fu7.5, $\left.0.9 \mathrm{mg} \mathrm{mL}^{-1}\right)$. In Figure $2 \mathrm{~B}$ it is possible to confirm the presence of fucoidan by observing the color change of the NFMs. Fucoidan in solution presents a light brown color, and the membranes with fucoidan are more yellowish than the controls, which represents its original white color. After toluidine blue staining (Figure 2C) it is clear that the NFM that contain fucoidan becomes purple, whereas the control is not stained.

3.3. Characterization of Bare and Fucoidan-Functionalized NFM. 3.3.1. Contact Angle. The hydrophilicity of NFM with or without immobilized fucoidan was determined by the water contact angle. In the table of Figure 3 it is possible to observe that, by increasing the concentration of immobilized fucoidan, NFMs presented a lower contact angle, being more hydrophilic. Electrospun NFMs without fucoidan presented a water contact angle of $121^{\circ} \pm 8$. On the other hand, NFMs with immobilized fucoidan (Fu10) were much more hydrophilic, presenting a water contact angle of $36^{\circ} \pm 8$.

3.3.2. XPS Analysis. XPS was used to analyze the surface chemistry of the different NFMs with or without immobilized fucoidan. From the quantitative analysis (Table 1) it was possible to observe that carbon and oxygen are present in all the NFMs with immobilized fucoidan, as well as in the control (without fucoidan), as expected since both elements are constituents of PCL and fucoidan chemical structures. With increasing fucoidan concentration for the functionalization of NFMs, there was a decrease in the atomic concentration of carbon and an increase of oxygen and, particularly, sulfur, which originated from the sulfate groups present on immobilized fucoidan.

A general spectrum of the control condition (Figure 4A) indicates the presence of two main peaks representing carbon $(291.40 \mathrm{eV})$ and oxygen (537.12 eV). For Ful0 (Figure 4B), three main peaks are detected: carbon $(297.70 \mathrm{eV})$, oxygen (544.47), and sulfur (176.0). After fucoidan immobilization, a
Table 1. Elemental Quantitative Analysis of the Surface of NFMs Functionalized with Different Fucoidan Concentrations

\begin{tabular}{clcccc}
$\mathrm{mg} \mathrm{mL}^{-1}$ & & $\begin{array}{c}\text { atomic concn } \\
(\%)\end{array}$ & $\begin{array}{c}\text { error } \\
(\%)\end{array}$ & $\begin{array}{c}\text { mass concn } \\
(\%)\end{array}$ & $\begin{array}{c}\text { error } \\
(\%)\end{array}$ \\
\hline CTR & S 2p & & & & \\
& O 1s & 24.03 & 0.38 & 29.65 & 0.44 \\
& C 1s & 75.97 & 0.38 & 70.35 & 0.44 \\
Fu2.5 & O 1s & 25.29 & 0.38 & 30.90 & 0.43 \\
& S 2p & 0.39 & 0.05 & 0.95 & 0.13 \\
& C 1s & 74.32 & 0.38 & 68.15 & 0.44 \\
Fu5 & O 1s & 28.98 & 0.49 & 34.89 & 0.54 \\
& S 2p & 0.60 & 0.07 & 1.44 & 0.18 \\
& C 1s & 70.43 & 0.50 & 63.66 & 0.56 \\
Fu7.5 & O 1s & 28.91 & 0.55 & 34.57 & 0.61 \\
& S 2p & 1.07 & 0.09 & 2.56 & 0.22 \\
& C 1s & 70.02 & 0.57 & 62.86 & 0.63 \\
Fu10 & O 1s & 36.14 & 0.50 & 42.10 & 0.53 \\
& S 2p & 1.41 & 0.09 & 3.28 & 0.21 \\
& C 1s & 62.46 & 0.51 & 54.62 & 0.54 \\
\hline
\end{tabular}

peak starting at $177.95 \mathrm{eV}$ and ending at $167.51 \mathrm{eV}$ appears, which is attributed to the sulfur element ( $S 2 p$ ) from the fucoidan chain, confirming fucoidan immobilization (Figure 4C).

3.4. Biological Response. 3.4.1. Cytotoxicity over Human Melanoma Cells. The viability of the melanoma cells was lower on the NFM_Fu condition than on the CTR from day 3 onward. At days 3 and 7, NFM_Fu induced a decrease in melanoma cell viability (Figure 5A). Specifically, at day 3 , around $70 \%$ of melanoma cells are viable, whereas at day 7 there is a viability of around $50 \%$ when compared with the CTR. These observations were corroborated by SEM micrographs (Figure 5B). Cells were able to colonize the control NFM, covering its surface by showing an increasing number of melanoma cells along the course of the 7 days. Oppositely, in the case of the NFM Fu condition, this colonization of the mesh was not observe $\bar{d}$.

3.4.2. Cytotoxicity over Human Keratinocytes. The immobilization of fucoidan on the NFM did not present cytotoxic effects over seeded keratinocytes (Figure 6A). No significant differences were observed between the CTR and the NFM_Fu for all time points. When analyzing the SEM micrographs, keratinocytes covered the NFM surfaces, 

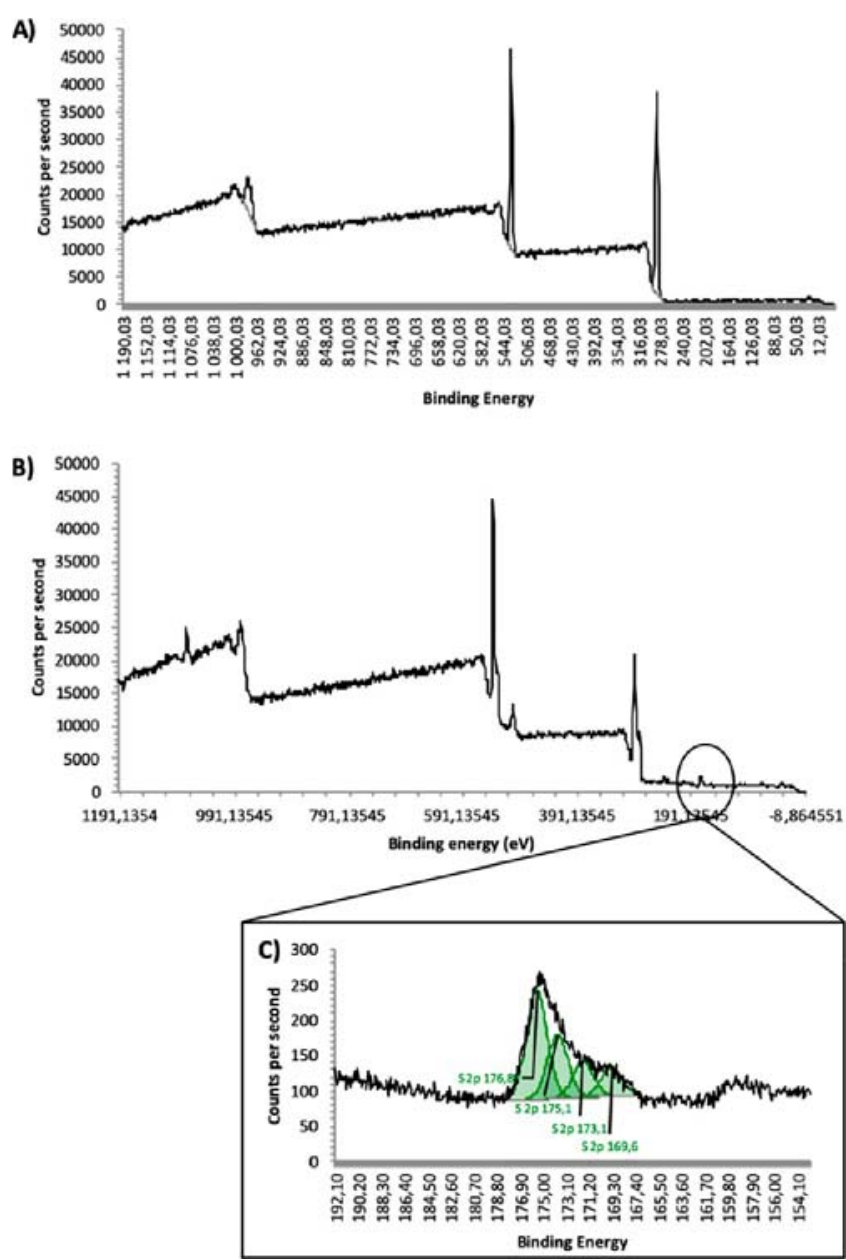

Figure 4. XPS analysis: survey spectrum of CTR (A); general spectra of Fu10 (B); and sulfur spectra of Fu10 (C).

presenting no morphological differences between the CTR and the NFM Fu conditions along the 7 days of culture.

3.4.3. Cytotoxicity over Human Dermal Fibroblasts. NFM Fu was also not cytotoxic for the fibroblasts (Figure 7). There were no significant differences between the CTR and the NFM_Fu regarding the viability for all time points. When analyzing the SEM micrographs, it is possible to observe that dermal fibroblasts covered the surface of the NFMs, forming a dense cell layer along time, and presenting no morphological differences between the two conditions.

\section{DISCUSSION}

Melanoma is considered one of the most aggressive and deadly skin cancers. ${ }^{17}$ If detected and treated at an early stage, melanoma presents a high survival rate. However, when melanoma is diagnosed in a late stage, the survival goes down to $15-20 \%$ at 5 years. ${ }^{15}$ Although there have been some recent advances in the field, there is still no effective therapeutic treatment for this type of cancer. Due to an increasing incidence of this cancer, the use of natural compounds, namely, the ones extracted from seaweeds, has been attracting a growing interest. It has been hypothesized that the consumption of brown seaweeds can be somehow related with the low incidence of melanoma in oriental countries. ${ }^{22,33}$ The best-known antitumor seaweed extract is fucoidan. Fucoidan in the soluble form has been reported to inhibit different types of cancer, including melanoma. ${ }^{22-24}$ However, due its natural origin and intrinsic variability, not all fucoidan extracts may present this antitumor behavior. ${ }^{7,27,34}$ Herein, as a first screening, fucoidan cytotoxicity and antitumor capability was tested in its soluble form. After this validation and trying to diminish the potential side effects of systemic therapies, an alternative and innovative therapeutic strategy is proposed by incorporating fucoidan on an electrospun nanofibrous mesh, aiming to act as a skin patch able to be easily and locally applied at the tumor site.

First, we evaluated the toxicity of fucoidan at different concentrations over different cell types. Besides testing with human melanoma cells (WM-115 cell line), primary human dermal fibroblasts and keratinocytes were also used as models for noncancer cells, since they are the main cellular components of skin dermis and epidermis, respectively. ${ }^{35}$ As described by others, soluble fucoidan is highly cytotoxic for melanoma cells. Fucoidan from different species cultured with melanoma cell lines showed a decrease in cell viability between 40 and $60 \%$, depending on the extracts, at $0.2 \mathrm{mg} \mathrm{mL}^{-1} \cdot{ }^{13} \mathrm{In}$ another study, the effects of native and oversulfated fucoidan were studied in tumor xenografts, where lung and melanoma cells were injected into mice. Both fucoidan extracts were able to reduce the tumor size after 21 days of daily injections. ${ }^{26}$ From our results, at $0.25 \mathrm{mg} \mathrm{mL}^{-1}$, a decrease in cells' viability starts to be observed. In addition, the viability of cancer cells was below $20 \%$ for concentrations of $0.5 \mathrm{mg} \mathrm{mL}^{-1}$ and above. For this same concentration of fucoidan $\left(0.5 \mathrm{mg} \mathrm{mL}^{-1}\right)$, keratinocytes presented a viability of around $60 \%$, whereas dermal fibroblasts present viabilities around $70 \%$. Even though fucoidan presents some cytotoxic effects over noncancer cells, fucoidan appears as a good candidate to treat melanoma.

Aiming to further decrease these cytotoxic side effects, the immobilization of fucoidan at the surface of nanofibrous meshes was proposed. Electrospun nanofibrous meshes are of special interest due to an easy functionalization, a high specific surface area and porosity, and a pore size that is smaller than the size of most cells. ${ }^{36,37}$ The production, activation, and insertion of amine groups at the surface of electrospun PCL nanofibrous meshes has been previously described and optimized by our group. ${ }^{28,29}$ Few studies have reported the use of nanofibrous-based systems to treat melanoma, namely, by the incorporation of chemotherapeutic drugs (i.e., doxorubicin and imiquimod). ${ }^{38,39}$ The use of fucoidan, a natural compound, has never been reported to be immobilized at the surface of electrospun nanofiber meshes. In fact, there are only a few reports where fucoidan has been combined with other polymers during the electrospinning process for tissue regeneration purposes. ${ }^{40-42}$

To quantify the immobilized fucoidan, Toluidine Blue assay was established. ${ }^{43,44}$ As it was possible to observe, control NFMs are not stained, whereas the ones with immobilized fucoidan are stained in blue, with increasing intensity with fucoidan content (data not shown). The system seems not to have reached its maximum immobilization capacity (maximum fucoidan concentration tested was $10 \mathrm{mg} \mathrm{mL}^{-1}$, which is the maximum solubility of fucoidan), but different initial concentrations lead to different percentages of immobilization.

The immobilization of fucoidan on electrospun NFMs was validated by contact angle and XPS analysis. As expected, the highest fucoidan concentrations presented the highest percentage of sulfur atoms, meaning that a higher concentration of fucoidan has been immobilized. Furthermore, the 

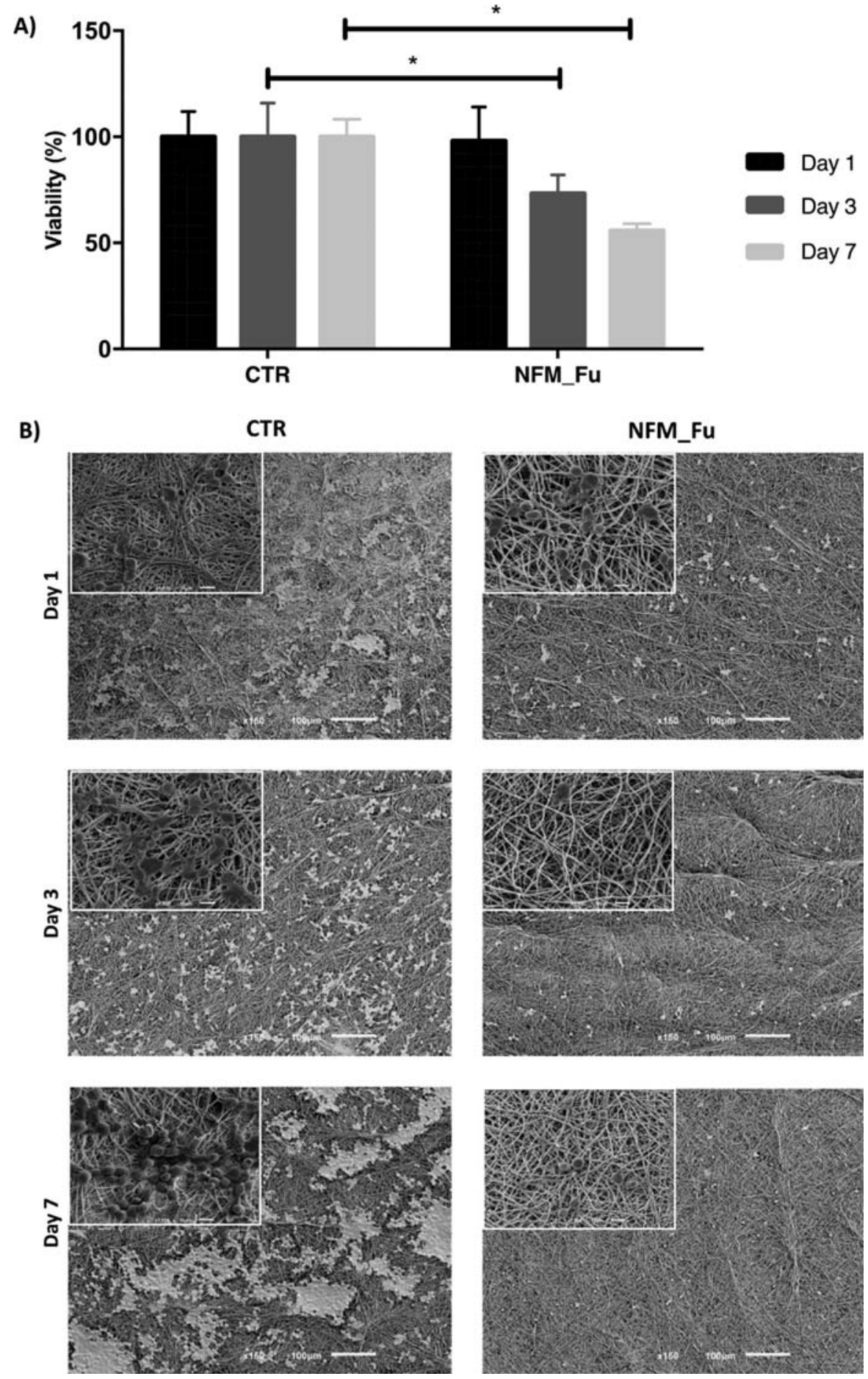

Figure 5. Cell viability (A) and representative SEM images (B) of human melanoma cells cultured on NFMs with and without (CTR) fucoidan. Data were considered statistically different if $p<0.05$.

immobilization of fucoidan also increased the oxygen content due to the presence of this element in functional groups within the polysaccharide structure, including the sulfates. ${ }^{45}$

One of the major disadvantages of some current cancer treatments is the fact that, besides affecting the tumor, they also have severe toxic effects over many healthy tissues. ${ }^{46}$ Therefore, when developing systems for melanoma treatment, there is the need to evaluate their cytotoxic effects not only over melanoma cells, but also over adjacent noncancer cells. The fucoidan-based system was tested over primary human keratinocytes (most common cell type in the epidermis) and fibroblasts (most common cell type in the dermis), as well as over a representative human melanoma cell line WM-115 (a cell line originated from the primary tumor with competence for metastasis) that has been used in different studies. ${ }^{47}$ This cell line features the specific V600D (Val600Asp) mutation at codon 600 in the BRAF gene. It also expresses PTEN loss of function, including hemizygous deletion, although wild-type for N-RAS, c-KIT, and CDK4 genes). It is known that tumors are highly heterogeneous and that different responses may be 

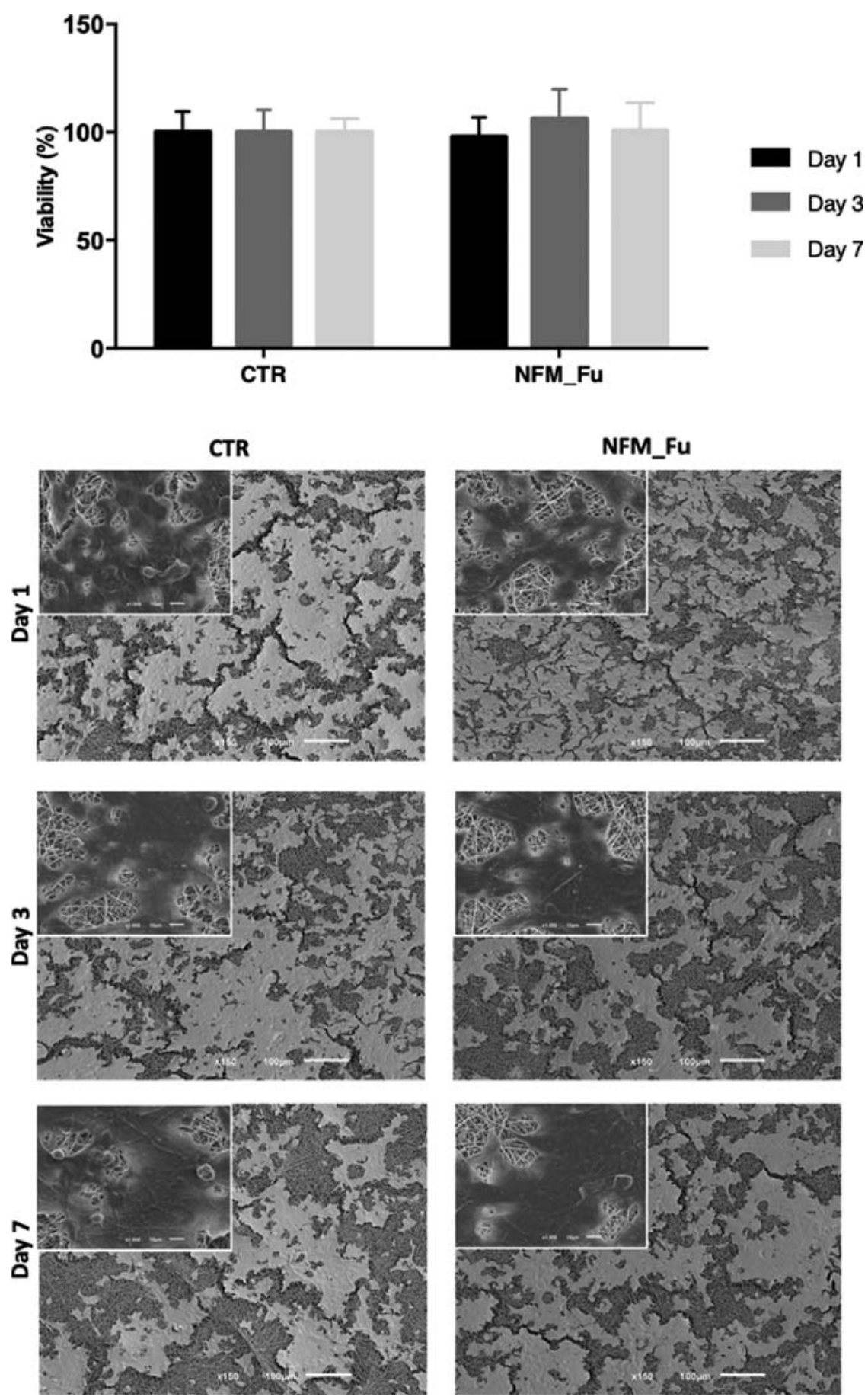

Figure 6. Cell viability (A) and representative SEM images (B) of human keratinocytes cultured on NFMs with and without (CTR) fucoidan and control NFMs. Data were considered statistically different if $p<0.05$.

observed by studying different cell lines. However, as a first in vitro validation of this system and by having in consideration the tumor microenvironment, valuable and promising results were achieved. In vitro results revealed a $50 \%$ decrease on melanoma cells' viability while maintaining the viability of normal cells, which is an indication of the selectiveness and effectiveness of the developed functionalized nanofibrous membranes. When compared to the cytotoxic effects of soluble fucoidan over the same cell types, fucoidan immobilized at the surface of NFMs decreased the cytotoxic effects over keratinocytes and dermal fibroblasts. These differences may be attributed to the fact that soluble fucoidan may be more bioactive, exerting an effective antitumor effect at lower concentrations than when immobilized. Therefore, fucoidan-based systems are not so cytotoxic to noncancer cells as soluble fucoidan, although still effective over melanoma cells.

To the best of our knowledge, despite few studies regarding fucoidan usage as a bioactive agent, there is only one fucoidanbased system reported in the literature that aims to treat melanoma. Specifically, fucoidan-based nanoparticles with an affinity to P-selectin and incorporating different chemotherapeutic drugs (i.e., Paclitaxel and Doxorubicin) presented increasing toxicity when compared with untargeted drugs and 

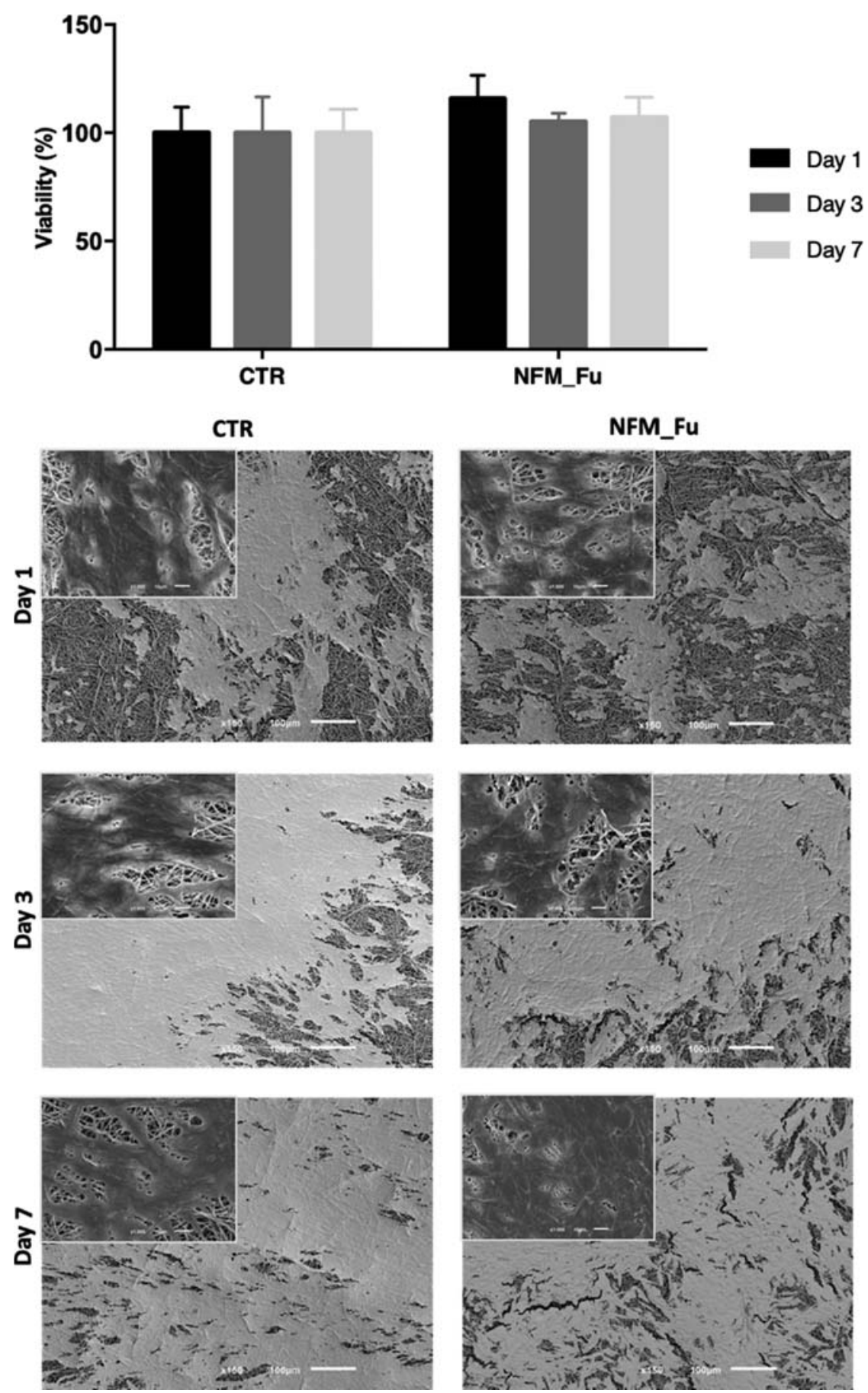

Figure 7. Cell viability (A) and representative SEM images (B) of human dermal fibroblasts (hDFs) cultured on NFMs with or without (CTR) fucoidan. Data were considered statistically different if $p<0.05$.

nanoparticles. ${ }^{48}$ Therefore, the proposed nanofibrous system represents a new and innovative concept that can be further explored for melanoma treatment, envisioning its use as an adjuvant system in the form of a patch applied after tumor excision.

\section{CONCLUSIONS}

Fucoidan, in its soluble form, presented antitumor activity potential since it presented increased toxicity to melanoma cells when compared with noncancer cells. Fucoidan was immobilized at the surface of NFM trying to develop a more local strategy to treat melanoma that can overcome some cytotoxic side effects. This novel fucoidan-based system presents decreased toxicity over human dermal fibroblasts and keratinocytes, although still toxic to melanoma cells. Therefore, it may be considered an adjuvant after tumor excision to tackle the eventual remaining melanoma cells. The proposed system appears as a potential effective therapeutic alternative that should be further validated for melanoma treatment. Furthermore, this therapy can be personalized according to the tumor characteristics by changing its size, shape, and treatment time. 


\section{AUTHOR INFORMATION}

\section{Corresponding Author}

Albino Martins - 3B's Research Group, I3Bs - Research Institute on Biomaterials, Biodegradables and Biomimetics, University of Minho, Headquarters of the European Institute of Excellence on Tissue Engineering and Regenerative Medicine, 4805-017 Barco, Guimarães, Portugal; ICVS/3B's - PT Government Associate Laboratory, Braga, Guimarães, Portugal; ○ orcid.org/0000-0003-3868-0251; Email: amartins@ i3bs.uminho.pt

\section{Authors}

Catarina Oliveira - 3B's Research Group, I3Bs - Research Institute on Biomaterials, Biodegradables and Biomimetics, University of Minho, Headquarters of the European Institute of Excellence on Tissue Engineering and Regenerative Medicine, 4805-017 Barco, Guimarães, Portugal; ICVS/3B's - PT Government Associate Laboratory, Braga, Guimarães, Portugal; (1) orcid.org/0000-0002-4014-6472

Ana I. Soares - 3B's Research Group, I3Bs - Research Institute on Biomaterials, Biodegradables and Biomimetics, University of Minho, Headquarters of the European Institute of Excellence on Tissue Engineering and Regenerative Medicine, 4805-017 Barco, Guimarães, Portugal; ICVS/3B's - PT Government Associate Laboratory, Braga, Guimarães, Portugal

Nuno M. Neves - 3B's Research Group, I3Bs - Research Institute on Biomaterials, Biodegradables and Biomimetics, University of Minho, Headquarters of the European Institute of Excellence on Tissue Engineering and Regenerative Medicine, 4805-017 Barco, Guimarães, Portugal; ICVS/3B's - PT Government Associate Laboratory, Braga, Guimarães, Portugal; The Discoveries Centre for Regenerative and Precision Medicine,

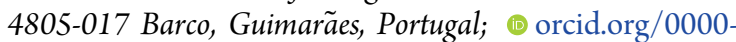
0003-3041-0687

Rui L. Reis - 3B's Research Group, I3Bs - Research Institute on Biomaterials, Biodegradables and Biomimetics, University of Minho, Headquarters of the European Institute of Excellence on Tissue Engineering and Regenerative Medicine, 4805-017 Barco, Guimarães, Portugal; ICVS/3B's - PT Government Associate Laboratory, Braga, Guimarães, Portugal; The Discoveries Centre for Regenerative and Precision Medicine, 4805-017 Barco, Guimarães, Portugal

Alexandra P. Marques - 3B's Research Group, I3Bs - Research Institute on Biomaterials, Biodegradables and Biomimetics, University of Minho, Headquarters of the European Institute of Excellence on Tissue Engineering and Regenerative Medicine, 4805-017 Barco, Guimarães, Portugal; ICVS/3B's - PT Government Associate Laboratory, Braga, Guimarães, Portugal; The Discoveries Centre for Regenerative and Precision Medicine, 4805-017 Barco, Guimarães, Portugal

Tiago H. Silva - 3B's Research Group, I3Bs - Research Institute on Biomaterials, Biodegradables and Biomimetics, University of Minho, Headquarters of the European Institute of Excellence on Tissue Engineering and Regenerative Medicine, 4805-017 Barco, Guimarães, Portugal; ICVS/3B's - PT Government Associate Laboratory, Braga, Guimarães, Portugal; 응 orcid.org/0000-0001-8520-603X

Complete contact information is available at: https://pubs.acs.org/10.1021/acs.biomac.0c00482

\section{Author Contributions}

The manuscript was written through contributions of all authors. All authors have given approval to the final version of the manuscript.

\section{Notes}

The authors declare no competing financial interest.

\section{ACKNOWLEDGMENTS}

This work was developed under the scope of the Structured Projects for R\&D\&I NORTE-01-0145-FEDER-000021 and NORTE-01-0145-FEDER-000023 supported by the Northern Portugal Regional Operational Programme (NORTE 2020), under the Portugal 2020 Partnership Agreement. The authors would like also to thank NORTE 2020 for financing the Ph.D. scholarship of C.O. "Norte-08-5369-000037" and the Portuguese Foundation for Science and Technology for the Investigator Grant of A.M. (IF/00376/2014). Dr. Luisa Rodrigues is acknowledged by the XPS analysis.

\section{REFERENCES}

(1) Arruebo, M.; Vilaboa, N.; Sáez-Gutierrez, B.; Lambea, J.; Tres, A.; Valladares, M.; González-Fernández, A. Assessment of the Evolution of Cancer Treatment Therapies. Cancers 2011, 3, 32793330.

(2) Cragg, G. M.; Newman, D. J. Natural products: a continuing source of novel drug leads. Biochim. Biophys. Acta, Gen. Subj. 2013, 1830, 3670-3695.

(3) de Jesus Raposo, M. F.; de Morais, A. M. B.; de Morais, R. M. S. C. Marine Polysaccharides from Algae with Potential Biomedical Applications. Mar. Drugs 2015, 13, 2967-3028.

(4) Silva, T. H.; Alves, A.; Popa, E. G.; Reys, L. L.; Gomes, M. E.; Sousa, R. A.; Silva, S. S.; Mano, J. F.; Reis, R. L. Marine algae sulfated polysaccharides for tissue engineering and drug delivery approaches. Biomatter 2012, 2, 278-289.

(5) Fitton, J. H.; Stringer, D. N.; Karpiniec, S. S. Therapies from Fucoidan: An Update. Mar. Drugs 2015, 13, 5920-5946.

(6) Ale, M. T.; Mikkelsen, J. D.; Meyer, A. S. Important Determinants for Fucoidan Bioactivity: A Critical Review of Structure-Function Relations and Extraction Methods for FucoseContaining Sulfated Polysaccharides from Brown Seaweeds. Mar. Drugs 2011, 9, 2106-2130.

(7) Oliveira, C.; Ferreira, A. S.; Novoa-Carballal, R.; Nunes, C.; Pashkuleva, I.; Neves, N. M.; Coimbra, M. A.; Reis, R. L.; Martins, A.; Silva, T. H. The Key Role of Sulfation and Branching on Fucoidan Antitumor Activity. Macromol. Biosci. 2017, 17, 1600340.

(8) Li, B.; Lu, F.; Wei, X.; Zhao, R. Fucoidan: Structure and Bioactivity. Molecules 2008, 13, 1671-1695.

(9) Wang, J.; Zhang, Q.; Zhang, Z.; Song, H.; Li, P. Potential antioxidant and anticoagulant capacity of low molecular weight fucoidan fractions extracted from Laminaria japonica. Int. J. Biol. Macromol. 2010, 46, 6-12.

(10) Abudabbus, A.; Badmus, J. A.; Shalaweh, S.; Bauer, R.; Hiss, D. Effects of Fucoidan and Chemotherapeutic Agent Combinations on Malignant and Non-malignant Breast Cell Lines. Curr. Pharm. Biotechnol. 2017, 18, 748-757.

(11) Alwarsamy, M.; Gooneratne, R.; Ravichandran, R. Effect of fucoidan from Turbinaria conoides on human lung adenocarcinoma epithelial (A549) cells. Carbohydr. Polym. 2016, 152, 207-213.

(12) Tsai, H.-L.; Tai, C.-J.; Huang, C.-W.; Chang, F.-R.; Wang, J.-Y. Efficacy of Low-Molecular-Weight Fucoidan as a Supplemental Therapy in Metastatic Colorectal Cancer Patients: A Double-Blind Randomized Controlled Trial. Mar. Drugs 2017, 15, 122.

(13) Vishchuk, O. S.; Ermakova, S. P.; Zvyagintseva, T. N. The fucoidans from brown algae of Far-Eastern seas: Anti-tumor activity and structure-function relationship. Food Chem. 2013, 141, 12111217. 
(14) Wang, Z.-J.; Zheng, L.; Yang, J.-M.; Kang, Y.; Park, Y.-D. Proteomic analyses for profiling regulated proteins/enzymes by Fucus vesiculosus fucoidan in B16 melanoma cells: A combination of enzyme kinetics functional study. Int. J. Biol. Macromol. 2018, 112, 667-674.

(15) American Cancer Society, https://www.cancer.org/cancer/ melanoma-skin-cancer.html, accessed 2018.

(16) Ferlay, J.; Soerjomataram, I.; Dikshit, R.; Eser, S.; Mathers, C.; Rebelo, M.; Parkin, D. M.; Forman, D.; Bray, F. Cancer incidence and mortality worldwide: sources, methods and major patterns in GLOBOCAN 2012. Int. J. Cancer 2015, 136, E359-86.

(17) Fitzmaurice, C.; Dicker, D.; et al. The Global Burden of Cancer 2013. JAMA Oncol 2015, 1, 505-527.

(18) World Health Organization, http://www.who.int/uv/faq/ skincancer/en/index1.html, accessed 2018.

(19) Bertolotto, C. Melanoma: From Melanocyte to Genetic Alterations and Clinical Options. Scientifica 2013, 2013, 635203.

(20) Batus, M.; Waheed, S.; Ruby, C.; Petersen, L.; Bines, S. D.; Kaufman, H. L. Optimal Management of Metastatic Melanoma: Current Strategies and Future Directions. Am. J. Clin. Dermatol. 2013, 14, 179-194.

(21) Widakowich, C.; de Castro, G.; de Azambuja, E.; Dinh, P.; Awada, A. Side effects of approved molecular targeted therapies in solid cancers. Oncologist 2007, 12 (12), 1443-55.

(22) Teas, J.; Irhimeh, M. R. Melanoma and brown seaweed: an integrative hypothesis. J. Appl. Phycol. 2017, 29, 941-948.

(23) Ale, M. T.; Maruyama, H.; Tamauchi, H.; Mikkelsen, J. D.; Meyer, A. S. Fucoidan from Sargassum sp. and Fucus vesiculosus reduces cell viability of lung carcinoma and melanoma cells in vitro and activates natural killer cells in mice in vivo. Int. J. Biol. Macromol. 2011, 49, 331-336.

(24) Ale, M. T.; Maruyama, H.; Tamauchi, H.; Mikkelsen, J. D.; Meyer, A. S. Fucose-containing sulfated polysaccharides from brown seaweeds inhibit proliferation of melanoma cells and induce apoptosis by activation of caspase-3 in vitro. Mar. Drugs 2011, 9, 2605-2621.

(25) Wang, Z.-J.; Xu, W.; Liang, J.-W.; Wang, C.-S.; Kang, Y. Effect of Fucoidan on B16 Murine Melanoma Cell Melanin Formation and Apoptosis. Afr. J. Tradit., Complementary Altern. Med. 2017, 14, 149155.

(26) Koyanagi, S.; Tanigawa, N.; Nakagawa, H.; Soeda, S.; Shimeno, $\mathrm{H}$. Oversulfation of fucoidan enhances its anti-angiogenic and antitumor activities. Biochem. Pharmacol. 2003, 65, 173-179.

(27) Dithmer, M.; Kirsch, A.-M.; Richert, E.; Fuchs, S.; Wang, F.; Schmidt, H.; Coupland, S. E.; Roider, J.; Klettner, A. Fucoidan Does Not Exert Anti-Tumorigenic Effects on Uveal Melanoma Cell Lines. Mar. Drugs 2017, 15, 193.

(28) Martins, A.; Pinho, E. D.; Faria, S.; Pashkuleva, I.; Marques, A. P.; Reis, R. L.; Neves, N. M. Surface Modification of Electrospun Polycaprolactone Nanofiber Meshes by Plasma Treatment to Enhance Biological Performance. Small 2009, 5, 1195-1206.

(29) Monteiro, N.; Martins, A.; Pires, R.; Faria, S.; Fonseca, N. A.; Moreira, J. N.; Reis, R. L.; Neves, N. M. Immobilization of bioactive factor-loaded liposomes on the surface of electrospun nanofibers targeting tissue engineering. Biomater. Sci. 2014, 2, 1195-1209.

(30) Kubaski, F.; Osago, H.; Mason, R. W.; Yamaguchi, S.; Kobayashi, H.; Tsuchiya, M.; Orii, T.; Tomatsu, S. Glycosaminoglycans detection methods: Applications of mass spectrometry. Mol. Genet. Metab. 2017, 120, 67-77.

(31) Taylor, A. Auger and X-ray photoelectron spectroscopy. J. Chem. Technol. Biotechnol. 1992, 53, 215-215.

(32) Costa, M.; Cerqueira, M. T.; Santos, T. C.; Sampaio-Marques, B.; Ludovico, P.; Marques, A. P.; Pirraco, R. P.; Reis, R. L. Cell sheet engineering using the stromal vascular fraction of adipose tissue as a vascularization strategy. Acta Biomater. 2017, 55, 131-143.

(33) Karimkhani, C.; Green, A. C.; Nijsten, T.; Weinstock, M. A.; Dellavalle, R. P.; Naghavi, M.; Fitzmaurice, C. The global burden of melanoma: results from the Global Burden of Disease Study 2015. Br. J. Dermatol. 2017, 177, 134-140.
(34) Oliveira, C.; Neves, N. M.; Reis, R. L.; Martins, A.; Silva, T. H. A review on fucoidan antitumor strategies: From a biological active agent to a structural component of fucoidan-based systems. Carbohydr. Polym. 2020, 239, 116131.

(35) Boer, M.; Duchnik, E.; Maleszka, R.; Marchlewicz, M. Structural and biophysical characteristics of human skin in maintaining proper epidermal barrier function. Postepy Dermatol Alergol. 2016, 1, 1-5.

(36) Zhan, J.; Singh, A.; Zhang, Z.; Huang, L.; Elisseeff, J. H. Multifunctional aliphatic polyester nanofibers for tissue engineering. Biomatter 2012, 2, 202-212.

(37) Oliveira, C.; Costa-Pinto, A. R.; Reis, R. L.; Martins, A.; Neves, N. M. Biofunctional Nanofibrous Substrate Comprising Immobilized Antibodies and Selective Binding of Autologous Growth Factors. Biomacromolecules 2014, 15, 2196-2205.

(38) Guo, M.; Zhou, G.; Liu, Z.; Liu, J.; Tang, J.; Xiao, Y.; Xu, W.; Liu, Y.; Chen, C. Direct site-specific treatment of skin cancer using doxorubicin-loaded nanofibrous membranes. Sci. Bull. 2018, 63, 92100.

(39) Lin, W.-C.; Yeh, I. T.; Niyama, E.; Huang, W.-R.; Ebara, M.; Wu, C.-S. Electrospun Poly $(\varepsilon$-caprolactone $)$ Nanofibrous Mesh for Imiquimod Delivery in Melanoma Therapy. Polymers 2018, 10, 231.

(40) Lee, J. S.; Jin, G. H.; Yeo, M. G.; Jang, C. H.; Lee, H.; Kim, G. $\mathrm{H}$. Fabrication of electrospun biocomposites comprising polycaprolactone/fucoidan for tissue regeneration. Carbohydr. Polym. 2012, 90, $181-188$.

(41) Shao, J.; Yu, N.; Kolwijck, E.; Wang, B.; Tan, K. W.; Jansen, J. A.; Walboomers, X. F.; Yang, F. Biological evaluation of silver nanoparticles incorporated into chitosan-based membranes. Nanomedicine (London, U. K.) 2017, 12, 2771-2785.

(42) Goonoo, N.; Bhaw-Luximon, A.; Jonas, U.; Jhurry, D.; Schönherr, H. Enhanced Differentiation of Human Preosteoblasts on Electrospun Blend Fiber Mats of Polydioxanone and Anionic Sulfated Polysaccharides. ACS Biomater. Sci. Eng. 2017, 3, 34473458.

(43) Sridharan, G.; Shankar, A. A. Toluidine blue: A review of its chemistry and clinical utility. Journal of Oral and Maxillofacial Pathology: JOMFP 2012, 16, 251-255.

(44) Jeong, J.-O.; Jeong, S. I.; Park, J.-S.; Gwon, H.-J.; Ahn, S.-J.; Shin, H.; Lee, J. Y.; Lim, Y.-M. Development and characterization of heparin-immobilized polycaprolactone nanofibrous scaffolds for tissue engineering using gamma-irradiation. RSC $A d v$. 2017, 7, 8963-8972.

(45) Ozaltin, K.; Lehocký, M.; Humpolíček, P.; Pelková, J.; Sáha, P. A New Route of Fucoidan Immobilization on Low Density Polyethylene and Its Blood Compatibility and Anticoagulation Activity. Int. J. Mol. Sci. 2016, 17, 908.

(46) Atashrazm, F.; Lowenthal, R. M.; Woods, G. M.; Holloway, A. F.; Dickinson, J. L. Fucoidan and Cancer: A Multifunctional Molecule with Anti-Tumor Potential. Mar. Drugs 2015, 13, 2327-2346.

(47) Vincent, K. M.; Postovit, L.-M. Investigating the utility of human melanoma cell lines as tumour models. Oncotarget 2017, 8, 10498-10509.

(48) Shamay, Y.; Elkabets, M.; Li, H.; Shah, J.; Brook, S.; Wang, F.; Adler, K.; Baut, E.; Scaltriti, M.; Jena, P. V.; Gardner, E. E.; Poirier, J. T.; Rudin, C. M.; Baselga, J.; Haimovitz-Friedman, A.; Heller, D. A. Pselectin is a nanotherapeutic delivery target in the tumor microenvironment. Sci. Transl. Med. 2016, 8, 345ra87. 